\title{
A solution for general exchange markets with indivisible goods when indifferences are allowed
}

\author{
Begoña Subiza $^{\dagger}$ and Josep E. Peris ${ }^{\dagger}$ \\ ${ }^{\dagger}$ Universitat d'Alacant, Dep. de Mètodes Quantitatius i Teoria Econòmica, \\ 03080 Alacant, Spain. (subiza@ua.es, peris@ua.es)
}

\begin{abstract}
It is well known that the core of an exchange market with indivisible goods is always non empty, although it may contain Pareto inefficient allocations. The strict core solves this shortcoming when indifferences are not allowed, but when agents' preferences are weak orders the strict core may be empty. On the other hand, when indifferences are allowed, the core or the strict core may fail to be stable sets, in the von Neumann and Morgenstern sense.

We introduce a new solution concept that improves the behaviour of the strict core, in the sense that it solves the emptiness problem of the strict core when indifferences are allowed in the individuals' preferences and whenever the strict core is non-empty, our solution is included on it. We define our proposal, the $M S$-set, by using a stability property (m-stability) that the strict core fulfills. Finally, we provide a min-max interpretation for this new solution.

Keywords: Indivisible goods, Exchange market, Strict core, Indifferences, $M S$-set, $m$-stability

JEL classification: C71; C78; D71; D78.
\end{abstract}

\section{Introduction.}

We consider the exchange market model with indivisible goods, started by the seminal paper of Shapley and Scarf (1974). There is a finite set of agents, each one owns one indivisible good and wants to exchange it for another, more preferred one. We assume the agents' preferences to be weak orders (agents 
may be indifferent between some goods). In such a market, the core (the set of undominated allocations) is always nonempty, but it may contain Pareto inefficient allocations. The strict core (the set of weak-undominated allocations, included in the core) solves this shortcoming, but whenever agents have some indifferences, it may be empty (Roth and Postlewaite, 1977).

It is well known that, when indifferences are allowed, the core (or the strict core) of exchange market model is not a stable set in the classical sense (Von Neumann and Morgenstern, 1944). In a recent paper (Peris and Subiza, 2013) a new stability notion, $m$-stability, has been introduced so that the core (and the strict core) is $m$-stable. We use this notion of stability in order to define a new solution concept, that we call $M S$-set. We prove that this set is always non-empty (even when indifferences in the agents' preferences are allowed) and is a selection of the strict core (whenever the strict core is non-empty). The $M S$-set is defined in a two-step process. First we select mstable sets with minimal cardinality and, secondly, we select those elements that are (directly or indirectly) better than as many allocations as possible. A min-max process providing the $M S$-set is also obtained.

\section{Preliminaries.}

\subsection{Binary relations.}

Given a finite set of alternatives $X$, a binary relation $\mathcal{P}$ defined on $X$ is said to be a preference relation if it is irreflexive: $\operatorname{not}(x \mathcal{P} x)$, for all $x$. The pair $(X, \mathcal{P})$ is often called a decision problem. From the preference relation $\mathcal{P}$, the transitive closure $\gg$ on $X$ is defined as usually:

$x \gg y \Leftrightarrow \exists x_{1}, x_{2}, \ldots, x_{k-1}, x_{k} \in X$ such that

$$
x=x_{1} \mathcal{P} x_{2}, \ldots, x_{i} \mathcal{P} x_{i+1}, \ldots, x_{k-1} \mathcal{P} x_{k}=y \text {. }
$$

\subsection{A market with indivisible goods (Shapley and Scarf, 1974).}

Let us consider an exchange market $\mathcal{M}$ with $n$ agents $N=\{1,2, \ldots, n\}$. Agent $i$ owns an indivisible object $w_{i}$, and has a preference relation $\mathcal{R}_{i}$ defined over the set of objects $\Omega=\left\{w_{1}, w_{2}, \ldots, w_{n}\right\}$. Throughout the work we consider that agents' preferences $\mathcal{R}_{i}$ are weak orders ${ }^{1}$ (indifferences are allowed). An allocation is any permutation of the initial endowment $\left(w_{1}, w_{2}, \ldots, w_{n}\right)$.

\footnotetext{
1 A weak order is a complete, reflexive and transitive binary relation. We will denote by $\mathcal{P}_{i}$ the strict preference (the asymmetric part of $\left.\mathcal{R}_{i}\right): x \mathcal{P}_{i} y$ iff $x \mathcal{R}_{i} y$ and $\operatorname{not}\left(y \mathcal{R}_{i} x\right) ; \mathcal{I}_{i}$ will denote the indifference relation: $x \mathcal{I}_{i} y$ iff $x \mathcal{R}_{i} y$ and $y \mathcal{R}_{i} x$.
} 
We will denote by $X$ the set of allocations. The dominance relation $\succ$ on the set of allocations $X$ is defined in the usual way: An allocation $x=\left(x_{1}, x_{2}, \ldots, x_{n}\right)$ dominates some other allocation $y=\left(y_{1}, y_{2}, \ldots, y_{n}\right)$, denoted by $x \succ y$, if there is some coalition $S \subseteq N$ such that

1. $\left\{x_{i}, i \in S\right\}=\left\{w_{i}, i \in S\right\}$; and

2. $x_{i} \mathcal{P}_{i} y_{i} \quad \forall i \in S$.

Shapley and Scarf (1974) proved that the core (the set of undominated allocations),

$$
\operatorname{co}(\mathcal{M})=\left\{x^{*} \in X: \quad \forall y \in X, \operatorname{not}\left(y \succ x^{*}\right)\right\},
$$

is always non-empty in this context. Nevertheless, it may contain Pareto inefficient allocations. The following example (Roth and Postlewaite, 1977) shows this fact.

Example 1. Let $\mathcal{M}_{1}$ be the market with three agents whose preferences are defined by the linear orders:

agent 1: $w_{3} \mathcal{P}_{1} w_{2} \mathcal{P}_{1} w_{1} ;$ agent 2: $w_{1} \mathcal{P}_{2} w_{2} \mathcal{P}_{2} w_{3} ;$ agent 3: $w_{2} \mathcal{P}_{3} w_{3} \mathcal{P}_{3} w_{1}$

Then, $\operatorname{co}\left(\mathcal{M}_{1}\right)=\left\{x \equiv\left(w_{3}, w_{1}, w_{2}\right), y \equiv\left(w_{2}, w_{1}, w_{3}\right)\right\}$. It is clear that the allocation $y$ is not Pareto efficient, since agents 1 and 3 are better off in the allocation $x$, whereas agent 2 gets the same object $\left(w_{1}\right)$ in both allocations.

To avoid this circumstance, a weak dominance relation $\succ_{w}$ on $X$ is introduced. An allocation $x=\left(x_{1}, x_{2}, \ldots, x_{n}\right)$ weak dominates some other allocation $y=\left(y_{1}, y_{2}, \ldots, y_{n}\right)$ if there is some coalition $S \subseteq N$ such that

1. $\left\{x_{i}, i \in S\right\}=\left\{w_{i}, i \in S\right\}$;

2. $x_{i} \mathcal{R}_{i} y_{i} \quad \forall i \in S$; and

3. $x_{k} \mathcal{P}_{k} y_{k}$ for some $k \in S$.

Following Quint and Wako (2004), we will name strict core, $\cos _{s}(\mathcal{M})$, the weak undominated allocations,

$$
\operatorname{co}_{s}(\mathcal{M})=\left\{x^{*} \in X: \quad \forall y \in X, \operatorname{not}\left(y \succ_{w} x^{*}\right)\right\}
$$

By definition, the strict core is included in the the core. In Example 1, $\operatorname{co}_{s}\left(\mathcal{M}_{1}\right)=\left\{x \equiv\left(w_{3}, w_{1}, w_{2}\right)\right\}$, which corresponds to the Pareto efficient allocation. It is clear, by definition of the weak dominance relation, that the 
allocations in the strict core are always Pareto efficient. Roth and Postlewaite (1977) proved that whenever the preferences of the agents are linear orders, the strict core is always non empty and contains a unique allocation. But this is not the case when indifferences in the individuals' preferences are allowed. In this situation, the strict core may be empty, or it may contain several allocations. $^{2}$ The following example (Quint and Wako, 2004) shows a case in which the strict core is empty.

Example 2. Let $\mathcal{M}_{2}$ be the market with three agents whose preferences are defined by the weak orders:

agent 1: $w_{2} \mathcal{P}_{1} w_{1} \mathcal{P}_{1} w_{3} ;$ agent 2: $w_{1} \mathcal{I}_{2} w_{3} \mathcal{P}_{2} w_{2} ;$ agent 3: $w_{2} \mathcal{P}_{3} w_{3} \mathcal{P}_{3} w_{1}$

The strict core is empty, whereas the core is

$$
\operatorname{co}\left(\mathcal{M}_{2}\right)=\left\{x \equiv\left(w_{1}, w_{3}, w_{2}\right), y \equiv\left(w_{2}, w_{1}, w_{3}\right)\right\}
$$

\section{Stability.}

The notion of stability (Von Neumann and Morgenstern, 1944) is stated as follows: given a decision problem $(X, \mathcal{P})$, a non-empty subset $V \subseteq X$ is called a stable set if

a) for all $x, y \in V, \operatorname{not}(x \mathcal{P} y)$; and

b) for all $y \in X \backslash V$ there is $x \in V$ such that $x \mathcal{P} y$.

Von Neumann and Morgenstern (1944) interpret this solution concept in terms of accepted standards of behavior, based on an internal stability condition ("no inner contradictions") and an external stability condition ("used to discredit non-conforming procedures"). Nevertheless, it is well known that the core (or the strict core) of an exchange market with indivisible goods may not be a stable set. In Peris and Subiza (2013) a new notion of stability is presented.

2 An interesting property (Wako (1991); see also Ma (1994)) is that, when the strict core contains several allocations, all of them are indifferent for all individuals; i.e., if $x$ and $y$ belong to the strict core, then $x_{i} \mathcal{I}_{i} y_{i}$ for all $i \in N$. So, when the strict core contains several allocations, it does not matter which one is selected. 
Definition 1. (Peris and Subiza, 2013) Given a decision problem $(X, \mathcal{P})$, a non-empty subset $V \subseteq X$ is an $\boldsymbol{m}$-stable set if

a) for all $x, y \in V$, whenever $x \gg y$ then $y \gg x$; and

b) for all $x, z \in X, x \in V, z \in X \backslash V$ implies not $(z \gg x)$.

It is proved that $m$-stable sets always exist in a finite context and that this stability condition is compatible with the core in the Shapley and Scarf model, in the sense that the core of an exchange market with indivisible goods is always an $m$-stable set with respect to the dominance relation, $\mathcal{P} \equiv \succ$, and the strict core, if non-empty, is an $m$-stable set with respect to the weak dominance relation, $\mathcal{P} \equiv \succ_{w}$.

\section{An $m$-stable solution concept.}

In order to chose a particular $m$-stable set we follow a two step process. Given a decision problem $(X, \mathcal{P})$, we define the set $M S(X, \mathcal{P})$ by:

Step 1: choose the m-stable sets with minimal cardinality ${ }^{3}$ and let $S$ be their union; and

Step 2: choose the elements $x \in S$ such that $|\{w \in X: x \gg w\}|$ is maximum.

Note that this set, when the set of alternatives is finite, is always nonempty. Now, we use the weak dominance relation $\mathcal{P} \equiv \succ_{w}$ in order to apply this notion to the context of exchange markets with indivisible goods.

Definition 2. Given an exchange market with indivisible goods $\mathcal{M}$

$$
M S(\mathcal{M})=M S\left(X, \succ_{w}\right)
$$

where $X$ is the set of allocations and $\succ_{w}$ is the weak dominance relation.

In order to illustrate this notion, we compute this set for the examples we have used before:

Example 1: $\quad M S\left(\mathcal{M}_{1}\right)=c o_{s}\left(\mathcal{M}_{1}\right)=\left\{\left(w_{3}, w_{1}, w_{2}\right)\right\} \varsubsetneqq c o\left(\mathcal{M}_{1}\right)$.

Example 2: $\quad M S\left(\mathcal{M}_{2}\right)=c o\left(\mathcal{M}_{2}\right)=\left\{\left(w_{1}, w_{3}, w_{2}\right),\left(w_{2}, w_{1}, w_{3}\right)\right\}$.

\footnotetext{
${ }^{3}$ For any finite set $A$, let us denote the cardinality of $A$ by $|A|$.
} 
The following result shows some properties of this set.

Theorem 1. Given an exchange market with indivisible goods $\mathcal{M}$,

a) $M S(\mathcal{M}) \neq \emptyset$.

b) $M S(\mathcal{M})$ is m-stable.

c) $\cos _{s}(\mathcal{M}) \neq \emptyset \Rightarrow M S(\mathcal{M}) \subseteq c o_{s}(\mathcal{M})$.

\section{Proof.}

a) We know (see Peris and Subiza (2013)) that, in this context, $m$-stable sets always exist. So, the process stated by steps 1 and 2 is always well defined and produces a non empty set.

b) In order to prove this part, we consider the following equivalence relation defined on $X$ :

$$
x \approx y \quad \Leftrightarrow \quad x=y \text { or }[x \gg y \text { and } y \gg x] .
$$

We denote the quotient set by $\mathbb{X}$, and $[x]$ will denote the class in $\mathbb{X}$ containing the element $x \in X$. We now define the following binary relation $\triangleright$ on $\mathbb{X}$ :

$$
[x],[y] \in \mathbb{X}, \quad[x] \triangleright[y] \Leftrightarrow[x] \neq[y] \text { and } \exists a \in[x], b \in[y]: a \gg b
$$

(see, for instance, Peris and Subiza (1994) for additional details about this relation and its properties). It is easy to observe that $m$-stable sets with minimal cardinality correspond to maximal classes in $(\mathbb{X}, \triangleright)$ with minimal cardinality, so step 1 selects some maximal classes. On the other hand, note that if some allocation $x$ is selected in step 2 then every allocation in its class must be selected. So, $M S(\mathcal{M})$ is union of $m$-stable sets and therefore $m$-stable (see Peris and Subiza (2013)).

$c$ ) If $c_{s}(\mathcal{M}) \neq \emptyset$, then an allocation $x^{*}$ is in the strict core if, and only if, $\left[x^{*}\right]=\left\{x^{*}\right\}$ is a maximal class in the quotient set $\mathbb{X}$. So $\left[x^{*}\right]$ is an $m$-stable set and the minimal cardinality of any $m$-stable set is 1 . Therefore, step 1 selects all the allocations in the strict core. Then, step 2 selects some of them.

In the following example we show that $M S(\mathcal{M})$ may be strictly contained in the strict core. 
Example 3. Let us consider an exchange marked with indivisible goods $\mathcal{M}$, with three people (six allocations), such that there are two weak undominated allocations, $x_{1}$ and $x_{2}$, and the relationships between these two allocations and the other ones is defined by:

$$
x_{1} \succ_{w} x_{3}, x_{1} \succ_{w} x_{4}, x_{1} \succ_{w} x_{5}, x_{1} \succ_{w} x_{6}, x_{2} \succ_{w} x_{3} .
$$

Then, $M S(\mathcal{M})=\left\{x_{1}\right\} \subsetneq c o_{s}(\mathcal{M})=\left\{x_{1}, x_{2}\right\}$.

\subsection{A min-max characterization.}

Given a decision problem $(X, \mathcal{P})$, we define the functions $s, g: X \rightarrow \mathbb{N}$,

$$
s(x)=|\{y \in X: y \gg x\}|, \quad g(x)=|\{y \in X: x \gg y\}| .
$$

Theorem 2. Given a decision problem $(X, \mathcal{P})$,

$$
M S(X, \mathcal{P})=\arg \max \{g(y), y \in \arg \min \{s(x), x \in X\}\} .
$$

Proof. The result is clear if we observe that if $[x]$ is a maximal class, $s(x)$ is the cardinality of such a maximal class. On the other hand, if $[z]$ is not a maximal class then $s(z)>s(x)$ for any maximal class $[x]$. Then, by minimizing function $s(x)$ we are selecting maximal classes (that is, $m$-stable sets) with minimal cardinality (note that the value $s(x)$ is the same for any element in a class). Finally, we choose those classes which maximize function $g(x)$, that is $M S(X, \mathcal{P})$.

Then, the set $M S(\mathcal{M})$ selects those allocations which are, directly or indirectly, weak dominated by as few elements as possible (minimal dominated) and, from these ones, those which are, directly or indirectly, better than as many allocations as possible (maximal dominant), following a min-max process.

Whenever individuals' preferences are linear orders (no indifferences are allowed), the strict core consists in just one allocation, that may be obtained throughout the Gale's Top Trading Cycles (TTC) algorithm (Shapley and Scarf, 1974). Our result in Theorem 2 provides an alternative way of finding this allocation, selecting the unique allocation $x^{*}$ satisfying $s\left(x^{*}\right)=0$. 


\section{References}

Ma, J., 1994. Strategy-proofness and the strict core in a market with indivisibilities. International Journal of Game Theory 23 (1), 75-83.

Peris, J. E., Subiza, B., 1994. Maximal elements of not necessarily acyclic binary relations. Economics Letters 44 (4), 385-388.

Peris, J. E., Subiza, B., 2013. A reformulation of von Neumann-Morgenstern stability: m-stability. Mathematical Social Sciences, forthcoming.

Quint, T., Wako, J., 2004. On houseswapping, the strict core, segmentation, and linear programming. Mathematics of Operations Research 29, 861877 .

Roth, A. E., Postlewaite, A., 1977. Weak versus strong domination in a market with indivisible goods. Journal of Mathematical Economics 4 (2), 131-137.

Shapley, L., Scarf, H., 1974. On cores and indivisibility. Journal of Mathematical Economics 1 (1), 23-37.

Von Neumann, J., Morgenstern, O., 1944. Theory of Games and Economic Behavior. Princeton: Princeton University Press.

Wako, J., 1991. Some properties of weak domination in an exchange market with indivisible goods. The Economic Studies Quarterly 42, 303-314. 